\title{
Visualization method of MR images useful for informed consent supported by augmented reality
}

\author{
Kaoru Kouzu \\ Department of Electrical Engineering, Faculty of Science and Engineering, \\ Kokushikan University, 4-28-1, Setagaya, Setagaya-ku, Tokyo, 154-8515, Japan \\ E-mail:kouzu@kokushikan.ac.jp
}

\begin{abstract}
In recent years, informed consent has become more and more important in every area of medical care. It is expected that new techniques for recognizing MR images will be developed. The Medical doctors look for indications of disease by using a color system in MR images and then they explain it to there patients. However, it is often difficult for patients to understand the explanations. In this case time, the use of AR (augmented reality) will be appropriate. Introducing this technique to patients will help then to recognize and understand two pictures of the MRI system. The method is based on the ideas proposed by our team of researchers.

The MR images give two kinds of weighted images: $\mathrm{T} 1$ and $\mathrm{T} 2$. We have developed a make the system which extracts tissue by comparing both weighted images. We can extract a specific tissue by setting the threshold value. The extracted tissues are mapped and superimposed over the original images. The AR contents are extracted from samples of water and fat. We are currently using software called the software "Metaio Creator". The image of the brain is the AR marker. The software recognizes the image and shows an image which is easier for the patient to understand. We have succeeded in creating a visualization of AR. We can see an image of the extracted sample by looking at the tablet. The brain image markers are displayed.
\end{abstract}

\section{Introduction}

In this paper, we will examine the visualization method for MR images that are is useful for informed consent and supported by AR (augmented reality).

In recent years, informed consent has been very important in every area of the medical scenes. The informed consent is carried out by medical doctors in the consultation room.

The doctors will discuss which areas are important for medical examination. However, it is difficult for a patient who has little knowledge of anatomy to understand the explanation given by a doctor using two-dimensional medical images. An example of this can be seen in MR images. The reason for this is that the images are often difficult to be recognize. In order to improve the patient's understanding, we must keep in mind that a doctor has a short time in which to give a patient an adeguate. Then, when a doctor explains the diagnostic results by using images, these techniques will help with the recognition of medical problems. We think that by of using the technique of AR, it will have a great benefit to patients. The technique of AR is popular these days, but it is used mainly in the field of entertainment.

In using this technique, patients will easily recognize the pictures. We use tablet PCs to look at AR contents and input medical records, because tablet PCs are rarely really used in the hospital these days. The method is based on the works proposed by our team of researchers. First of all, T1 and T2 weighted images are taken by using MRI. The extraction of tissue is enabled by comparing the grayscale levels of MR images.

We put an extracted images on the original MR images as the baseline date base and adjust the position. The next step, is to we make AR images by using software. The MR images, which can be seen on a tablet, can be displayed instantly. Then we make an animation sequentially from extracted tissue images. We can confirm MR images better when we use this system. Medical information may feel more familiar if we use these AR techniques. We hope that we will play an active part at medical institutions. 


\section{Internal Body Tissue Extraction Method}

\subsection{Weighted image of the MRI}

We get two kinds of sliced images T1W (T1 weighted image) and T2W (T2 weighted image). We use an MRI machine AIRIS2 manufactured by Hitachi medical corporation $^{[1]}$ shown in figure 1 . A magnetic field of $0.3 \mathrm{~T}$ is used to take images. The MR images are taken in the form of DICOM. DICOME stands for Digital Imaging and Communication in Medicine, which usually uses the format of CT, CR, and MRI, is that of a standard that defines a communication protocol between medical imaging equipment to deal with those images. Because the size of the file is big, the DICOM form uses jpeg to lower the computing speed. We convert them into JPG format using PC.

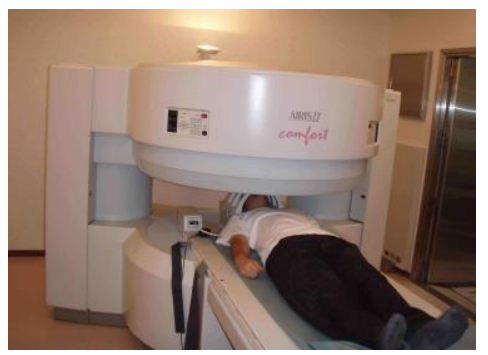

Fig. 1 MRI system

There are three kinds of photography directions of the MRI, as shown in figure2. They are transverse, sagittal, and coronal. Figure 3 is the sample taken from the coronal view.

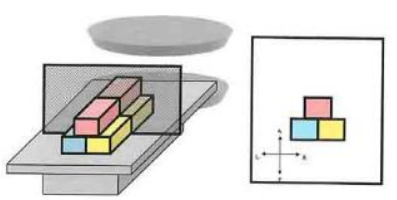

Transverse

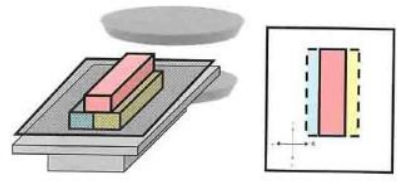

Coronal
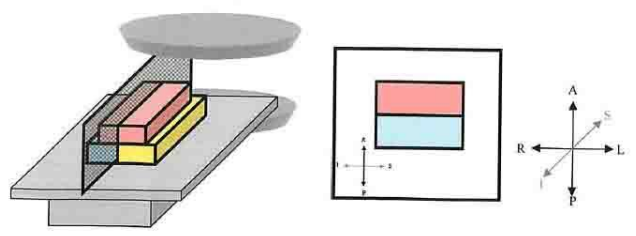

A: Anterior

P: Posterior

R: Right

L: Left

S: Superior

I: Inferior

Saggital

Fig. 2 Direction of the MRI

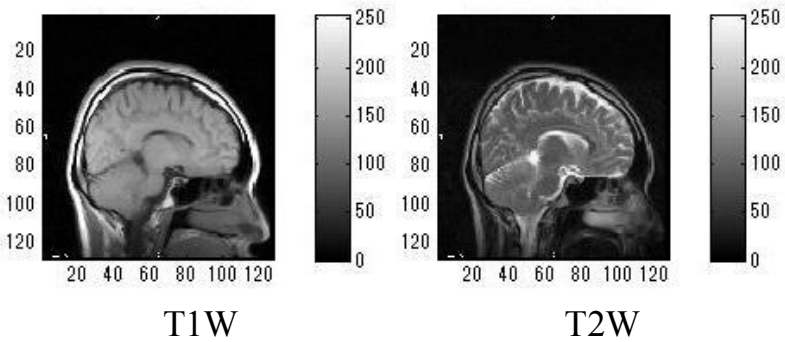

Fig. 3 Weighted image of MR

The MR image time depends on the number of sheets. To shoot 16 sheets for $\mathrm{T} 1$ and $\mathrm{T} 2$ respectively, it takes about 30 minutes. The bar beside the brain image shows a gray scale. The vertical and horizontal axes of the figure are a coordinate.

The classification of the tissue for some sample tissues are shown in table 1 . The data based on table 1 and table 2 are written in "MRI frrdom." [2]

Water is colored black, and fat is colored white according to $\mathrm{T} 1 \mathrm{~W}$. The relation between the combination of colors and pathological changes are described by the pattern in table 2 .

Table 1 Power strength of the tissue

\begin{tabular}{|c|c|c|}
\hline & T1W & T2W \\
\hline much water & low signal & high signal \\
\hline little water & low signal & low signal \\
\hline Fat & high signal & high signal \\
\hline
\end{tabular}

Table 2 Possibilities of disease assumed by the grayscale levels of MR images

\begin{tabular}{|c|c|c|}
\hline $\mathrm{T} 2 \mathrm{~W}$ & $\mathrm{~T} 1 \mathrm{~W}$ & $\begin{array}{c}\text { Possibility of disease in } \\
\text { the MR images }\end{array}$ \\
\hline & & $\begin{array}{l}\text { A benign tumor } \\
\text { An acute inflammation }\end{array}$ \\
\hline & & $\begin{array}{l}\text { A malignant tumor } \\
\text { Chronic inflammation }\end{array}$ \\
\hline & & $\begin{array}{l}\text { High protein liquid } \\
\text { Calcification }\end{array}$ \\
\hline
\end{tabular}

\subsection{Method of tissue extraction}

We use the MATLAB software for tissue extract.

We calculate the following histogram using an image processing program from MR images from figure 3, 
which is further revealed in figure 4 . The vertical axis represents is the number of pixels, while the horizontal axis represents is the gray scale value.

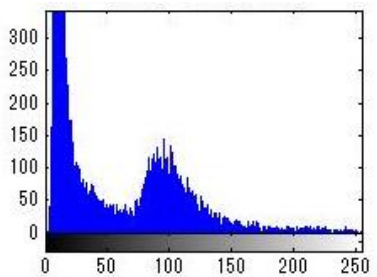

$\mathrm{T} 1 \mathrm{~W}$

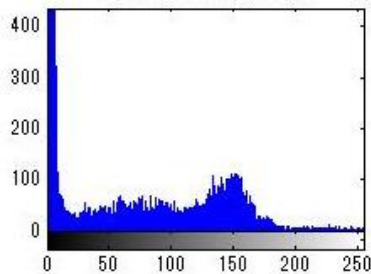

$\mathrm{T} 2 \mathrm{~W}$
Fig. 4 Histogram of MRI

The area of the tissue is made possible by comparing the grayscaled levels of MR images. For example, from the difference in gray scale, we can see extracted malignant tumors. We can select images of the tissue using binary images. We have calculated the values of the grayscale. In this paper, we have used the example of water and fat because they are easy to explain. We paint the tissue in different colors to distingwish each part. The water content is painted in blue, and fat content is painted in yellow.

In figure 5 , the threshold value of binarization as a value of water in image $\mathrm{T} 2 \mathrm{~W}$ is $150-256$, whereas $\mathrm{T} 1 \mathrm{~W}$ is 0 150 (Shown in blue), and the value of fat in T2W is 150 256 and T1W is 150-256 (Shown in orage). By changing the threshold value of the data represented by blue and orange, it is possible to extract a variety of tissues, we put an extracted image on the original MRI as the baseline date and adjusted the position, as shown in figure 6 .
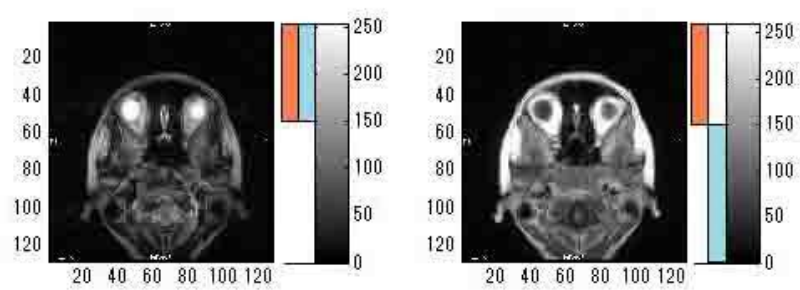

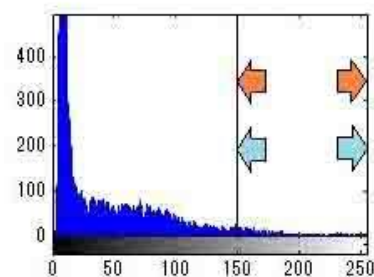

$\mathrm{T} 2 \mathrm{~W}$

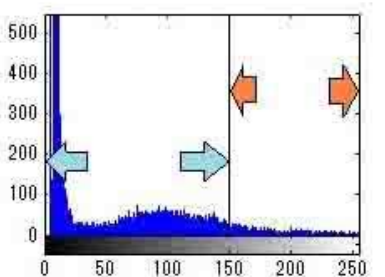

$\mathrm{T} 1 \mathrm{~W}$
Fig. 5 Value of the histogram

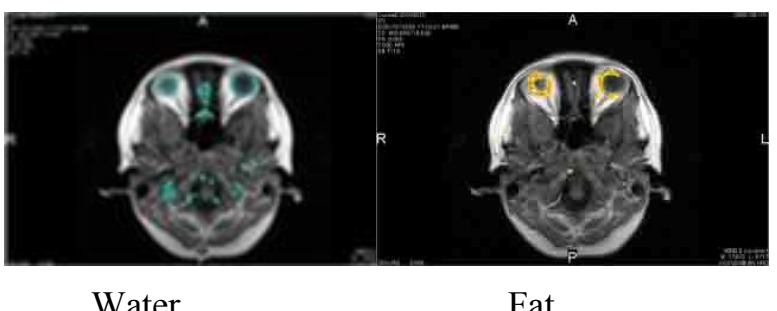

Fig. 6 Composite image

We overlay each tissue image on the original image, as shown in figure 7.

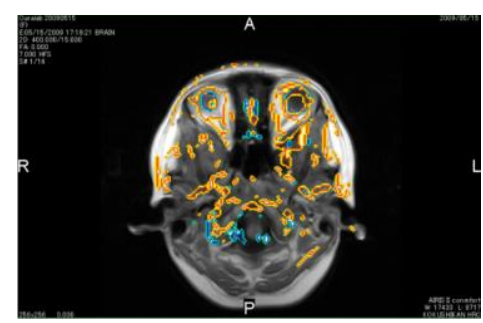

Fig. 7 Composite image

\subsection{Past studies of view point}

We studied other $3 \mathrm{D}$ expression methods ${ }^{[3][4]}$ before the study using this tablet.

To explain this image briefly, we create a 3D reconstruction of the images considering the viewpoint of the operator and their relationship to the subject, as shown in figure 8 . This relationship position diagram shows the viewpoint position.

We propose a system that focuses on the viewpoint of the operator. Our system creates a reconstructed image that shows the correct angle of the subject as seen by the operator and its line of sight. We measure each length, and calculate some parameters.

$$
\begin{gathered}
r=\sqrt{s^{2}+a^{2}} \\
\rho=\sqrt{r^{2}+h^{2}} \\
\alpha=\tan ^{-1} \frac{a}{s} \\
\beta=\tan ^{-1} \frac{h}{r}
\end{gathered}
$$

If the operator moves, the reconstructed image changes, and it becomes easy understand intuitively. We viewed the image using a polarized lens, which allowed us look at the image in stereoscopic vision. The projection method uses two small projectors to display the images in the doctor's office, as shown in figure 9. Therefore, it 
does not cost a large amount of money. In addition, we display composite coronal and transverse images of the brain. Then, we are able to get more detailed images.

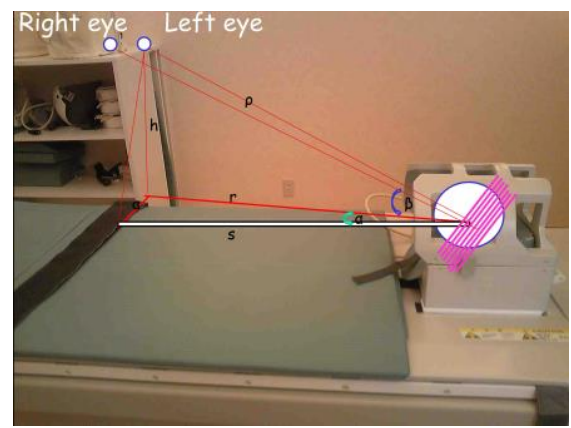

Fig. 8 Relation between subject and view point

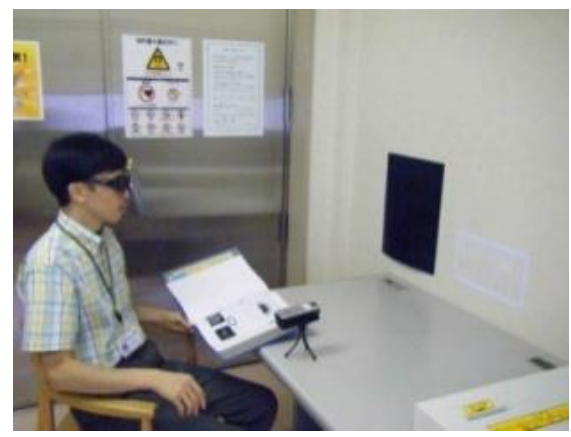

Fig. 9 The projection using the polarized lens

As described above, this is a good system to view $3 \mathrm{D}$. This is an example of how we used visualization to help achieve informed consent. We used one display in the examination room using AR.

\section{Visualizatin using AR technique}

\subsection{Making AR contents}

We use the software for "Metaio Creator" and "junaio" that are free to download. We show the procedure for making the contents in figure 10 .

We can create a QR code for the display in AR. This allow us to read it. On top of the baseline data of the brain image, this is an example of how extracted tissue is superimposed.

Figure 11 shows the region of extracted water and figure 12 is a screenshot from of "Metaio Creator". Figure 13 shows a sample of the visualization on the tablet using the AR program. Here, the image of the brain is the AR marker. The doctor will explain how to use the tablet to understand the superimposed images of the tissues in AR.
The software recognizes the image for patient and makes it easier to look at.

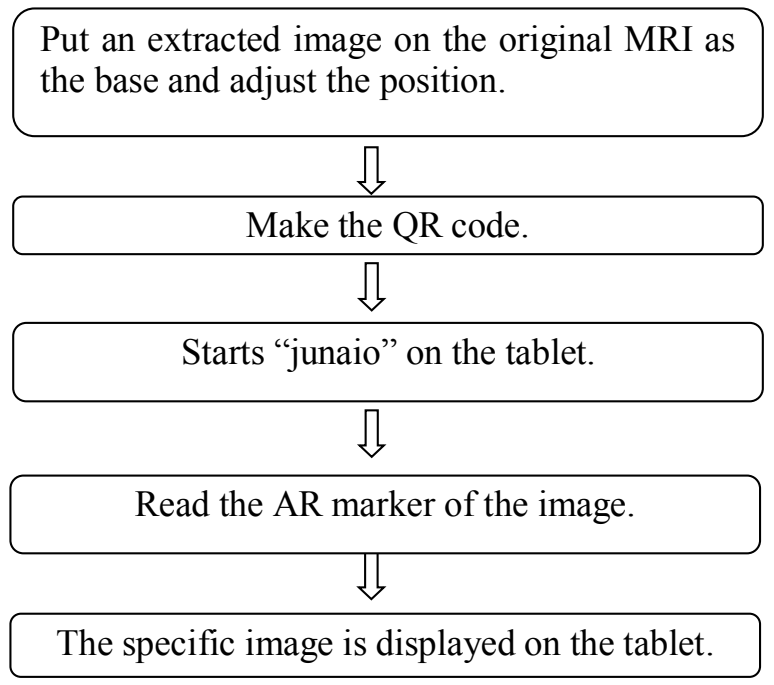

Fig. 10 Algorithm for making AR

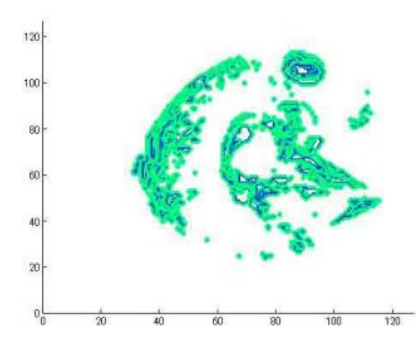

Fig. 11 Extracted water

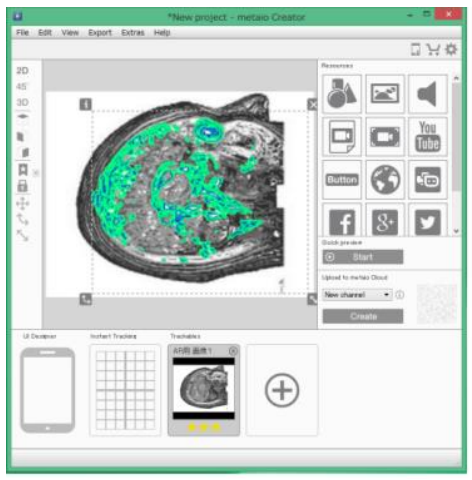

Fig. 12 Screenshot of "Metaio Creator"

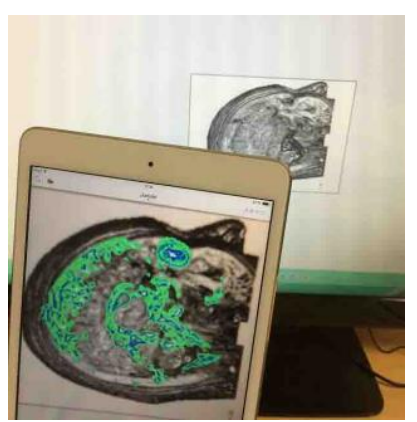

Fig. 13 The result of the algorithm 


\subsection{Adjustment of the position}

When we display AR contents, we can make a superimposed image that gives a feeling of depth. We make an extracted image that floats above the original image. The extracted image can be moved in the direction of a blue arrow according to the procedure, as shown in figure 14.

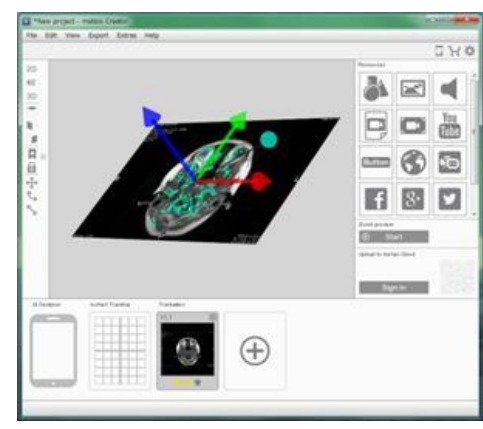

Fig. 14 Composite image

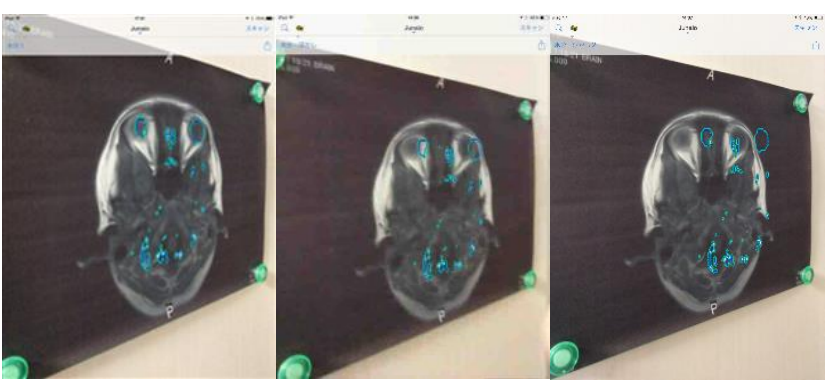

Fig. 15 The result of three diffrernt changes in depth

We can give the image depth by following these steps. The result is shown in figure 15. Figure 15 shows three AR images with different changes in depth. The depth of each image is increased from left to right.

As a result, when we look at a tablet from the direction which is not the front, it seems that the extracted image has shifted. It is preferable to let the tablet image connect with the original image in order to display AR correctly.

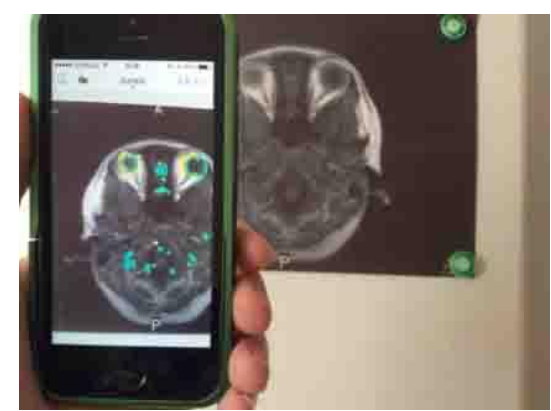

Fig.16 The result of the AR with a mobile phone
Figure 16 lets us view an original image and an extracted image together. The brain image behind the figure is the AR marker.

\subsection{Making the animation for tissue}

We take photographs of 16 sliced MR images. We make the animation by putting the sliced images of the water tissue together sequentially. Therefore we able to calculate through image processing how to extract water tissue from 16 sheets of MR images, by the method described above.

We perform image processing with an extracted water image of both $\mathrm{T} 1 \mathrm{~W}$ and $\mathrm{T} 2 \mathrm{~W}$, which gives us the result shown in figure17. We display the extracted image of the AR content in an animation, as shown in figure 18 .

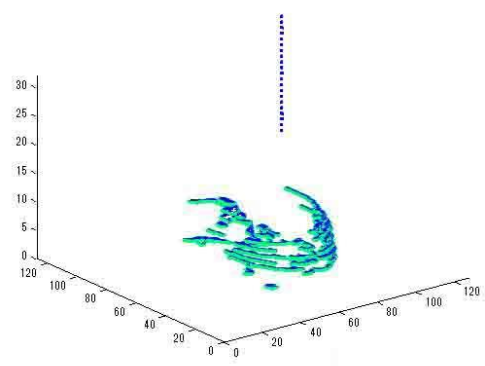

Fig.17 Tissue of 3-D

In the system, the animation and the photographed images both use a standard background.

The animation includes more information than a still image. It is thought to be an effective way to support informed consent when it is shown to the patient.
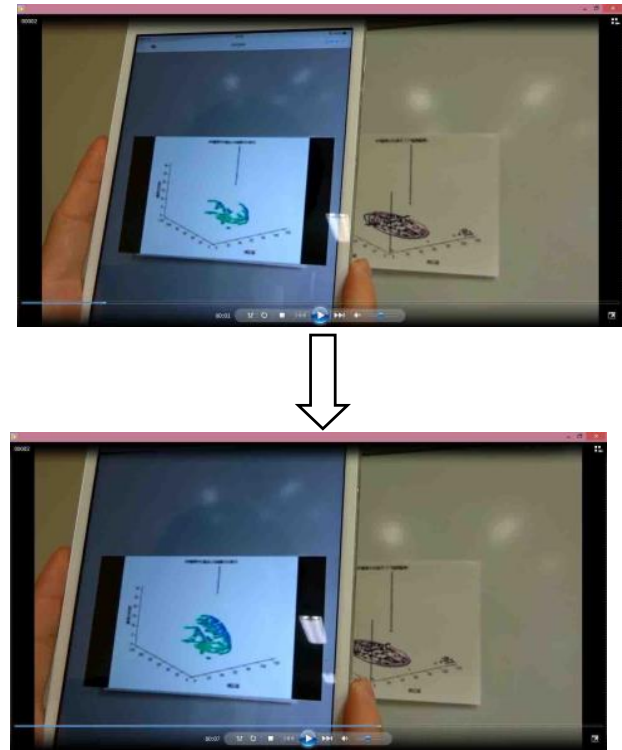

Fig. 18 A picture of the animation 


\section{Conclusions}

Though the use of image processing, we can successfully create a system that can be used for the processing of tissue extract images. We are able to view images of extracted water and fat with the AR system. We think that this system will enable us to detect diseases more easily. The use of AR is a technique used in various fields to connect reality with digital space. This paper emphasizes the practical use of AR using a tablet or mobile phone, and its future application in hospitals. Our previous studies have presented the visualization method for MR images using the stereoscopic 3D effect. This includes the use of a polarized lens and the anaglyph method, which requires a proper environment for the use of two projectors. Although this technology is a good idea as a stereoscopic vision, it is necessary to have the appropriate equipment and environment. How to use this tablet, where necessary without installing the projector can be inexpensively used because cost only tablets and software. Due to the high cost of installing a projector, we give instructions on how to use a tablet and the software.

Further evaluation and feedback is expected from medical professionals.

\section{References}

[1] I-MR model AIRIS- II , Made "Hitachi medical corpration", magnetic field $0.3 \mathrm{~T}$, power capacity max $5 \mathrm{~kW}$, average $500 \mathrm{~W}$.

[2] T. Takahara: MRI freedom (in Japanese), MEDICAL VIEW CO., 2008.

[3] K.Kouzu, K.Oura: A study on tissue extraction from magnetic resonance images and reconstruction of objects considering a view point, Annual Meeting of I. E. E. Japan, p.128, 2010.

[4] K.Kouzu, K. Oura: The superposition tissueextraction solid 3D expression method using the brain images of Magnetic Resonance, Annual Meeting of I. E. E. p. 135, Japan,2012.

[5] Hongen Liao, Takashi Inomata, Ichiro Sakuma: 3-D Augmented Reality for MRI-Guided Surgery Using Integral Videography AutostereoscopicImage Overlay, IEEE, TRANSACTIONS ON BIOMEDICAL ENGINEERING,VOL.57,NO.6.JUNE，pp.1476-1486, 2010 